\title{
Pseudoxanthoma Elasticum and Cardiorenal Disease: A Case Report
}

\author{
Luis D'Marco $^{1,2}$, Carlos Soto ${ }^{1}$, Fabiola Dapena ${ }^{1}$ \\ ${ }^{1}$ Consorci Sanitari del Alt Penedes-Garraf, Nephrology Department, Barcelona, Spain \\ ${ }^{2}$ Hospital Clínico Universitario, INCLIVA, Nephrology Department, Valencia, Spain
}

Received: 24/08/2019

Accepted: $23 / 11 / 2019$

Published: $17 / 12 / 2019$

How to cite this article: D'Marco L, Soto C, Dapena F. Pseudoxantoma elasticum and cardiorenal disease: a case report. EJCRIM 2019;7: doi:10.12890/2019_001260.

Conflicts of Interests: The Authors declare that there are no competing interest

Acknowledgements: The Authors thank Maria Romero-Parra for her help.

This article is licensed under a Commons Attribution Non-Commercial 4.0 License

\section{ABSTRACT}

Pseudoxanthoma elasticum (PXE) is a rare genetic disorder characterized by calcification of elastic fibres, skin lesions, fundus lesions and systemic vascular complications. PXE affects approximately 1 in 160,000 people, typically appearing as a formation of yellow papules containing abnormally calcified elastic fibres. The renal involvement of PXE has been reported. Several factors are known to promote soft tissue and accelerated arterial calcification in chronic kidney disease, including systemic inflammation, altered calcium and phosphate homeostasis, hypertension and a deficiency of endogenous calcification inhibitors. Given the impact of this disease, nephrologists may seek additional supportive features to improve and avoid the risk of complications. Moreover, PXE per se represents an interesting model to evaluate vascular disease in the early stages of renal disease.

\section{LEARNING POINTS}

- Pseudoxanthoma elasticum represents a rare disease that can involve renal problems.

- Vascular compromise in patients affected by pseudoxanthoma elasticum shares some components observed in patients with chronic kidney disease.

- Disorders of mineral and bone metabolism may coexist or be hidden in patients with pseudoxanthoma elasticum.

\section{KEYWORDS}

Pseudoxanthoma, chronic kidney disease, cardiorenal

\section{INTRODUCTION}

Pseudoxanthoma elasticum (PXE) is a rare genetic disorder caused by mutations in the ABCC6 gene, which is characterized by calcification of elastic fibres, skin lesions, fundus lesions and systemic cardiovascular complications, including coronary artery lesions, lower-leg arterial lesions and gastrointestinal bleeding ${ }^{[1]}$. The mode of inheritance in most families is autosomal recessive, but PXE with autosomal dominant inheritance has also been reported. PXE affects approximately 1 in 160,000 people, typically appearing as a formation of yellow papules containing abnormally calcified elastic fibres. Cutaneous lesions are seen in the neck, axilla, cubital fossa, inguinal region and periumbilical area. Angioid streaks are typically seen in the fundus due to breaks in Bruch's membrane. Thus, PXE represents a model disease for arteriosclerosis as it is thought to encompass an isolated arteriosclerotic type of arterial wall disease with relatively little interference by atherosclerotic arterial wall disease. Patients with PXE have low levels of inorganic pyrophosphate, a strong inhibitor of ectopic mineralization. This leads to a typical pattern of progressive calcification of elastic fibres in the skin, eyes and vasculature ${ }^{[2]}$.

The renal involvement of PXE has been reported. Several factors are known to promote soft tissue and accelerated arterial calcification in chronic kidney disease (CKD), including systemic inflammation, altered calcium and phosphate homeostasis, hypertension and a deficiency 
of endogenous calcification inhibitors. Lau et al. reported for the first time that CKD results in acquired ABCC6 transporter deficiency which may contribute to CKD-associated vascular and soft tissue calcification ${ }^{[3]}$. On the contrary, although calciphylaxis most often occurs in patients with end-stage renal disease (ESRD) and secondary hyperparathyroidism, non-uremic calciphylaxis has been described in PXEaffected patients without several forms of CKD.

\section{CASE DESCRIPTION}

A 77-year-old white Spanish woman referred to our outpatient clinic with hypertension and albuminuria with a normal glomerular filtration rate (GFR). In addition, she had a history of PXE since 1997, progressive visual deficiency, chronic left lower extremity oedema and an ischaemic stroke 4 years previously. She had primary hyperparathyroidism as a recent diagnostic. None of the patient's first-degree relatives had a history of PXE.

The physical examination showed a weight of $61 \mathrm{~kg}$; a height of $1.71 \mathrm{~m}$; BMl of $20.8 \mathrm{~kg} / \mathrm{m}^{2}$; blood pressure at $160 / 90 \mathrm{mmHg}$. Multiple, discrete, symmetrical, small yellow plaques as leathery skin were present bilaterally in the axillae, inguinal region and the neck. Angioid streaks were seen in the fundus. Asymmetric left lower extremity oedema was observed.

Laboratory findings were significant for an elevated parathyroid hormone level (170 pg/ml, reference range: $10-65 \mathrm{pg} / \mathrm{ml})$; low serum phosphorus ( $2.3 \mathrm{mg} / \mathrm{dl}$, reference range: $2.7-4.5 \mathrm{mg} / \mathrm{dl}$ ); hypercalcaemia (11.6 mg/dl, reference range: $8.5-10.5 \mathrm{mg} / \mathrm{dl}$ ) with a calciumphosphorous product of $26.6 \mathrm{mg} / \mathrm{dl}$; calciuria $376 \mathrm{mg} / 24 \mathrm{~h}$ and albuminuria $132 \mathrm{mg} / \mathrm{g}$. The remainder of the blood test results were normal (Hb $13.7 \mathrm{~g} / \mathrm{dl}$; Hto 43\%; fasting glucose $99 \mathrm{mg} / \mathrm{dl}$; uric acid $4.0 \mathrm{mg} / \mathrm{dl}$; total cholesterol $200 \mathrm{mg} / \mathrm{dl}$; TG $100 \mathrm{mg} / \mathrm{dl}$; creatinine $0.6 \mathrm{mg} / \mathrm{dl}$; eGFR < $90 \mathrm{ml} / \mathrm{min}$; K $4.7 \mathrm{meq} / \mathrm{l}$; Na $131 \mathrm{meq} / \mathrm{l} ; \mathrm{Cl} 107 \mathrm{meq} / \mathrm{l}$; calcitriol 46 pg/ml.

Imaging tests showed kidney ultrasound imaging with a slight increase in the echogenicity of the renal parenchyma (Fig. 1) and normal BMD (Fig. 2).
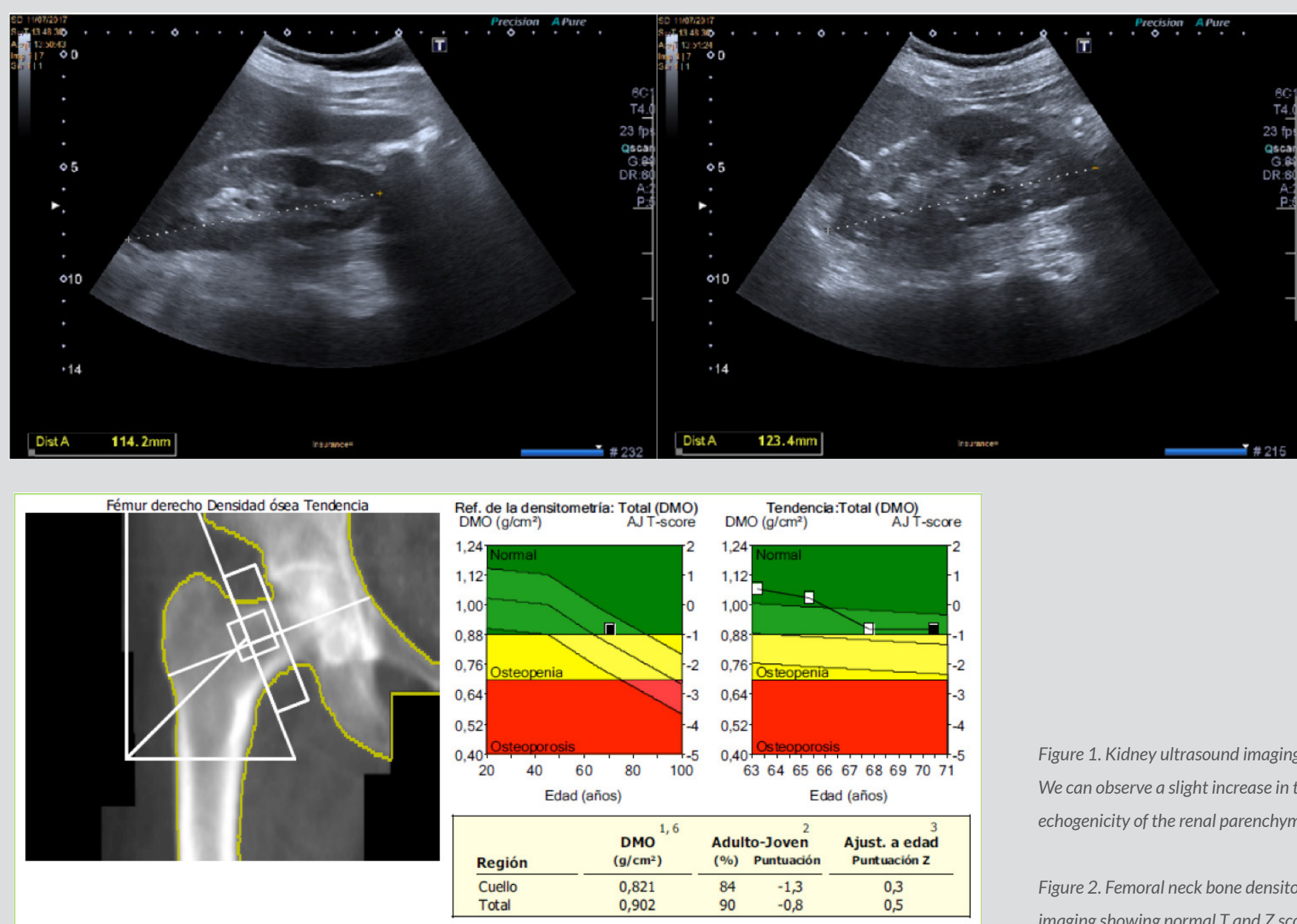

Figure 1. Kidney ultrasound imaging. We can observe a slight increase in the echogenicity of the renal parenchyma

Figure 2. Femoral neck bone densitometry imaging showing normal $T$ and $Z$ scores 
Treatment

The patient was taking: losartan 50 mg BID, acetylsalicylic acid 100 mg OD, simvastatin 20 mg OD, omeprazole 20 mg OD, pregabalin 25 mg OD.

\section{DISCUSSION}

As mentioned earlier, PXE has a high prevalence of vascular disease (in particular, peripheral arterial disease and cerebrovascular disease), which is thought to be caused by the proposed specific arteriosclerotic arterial wall changes occurring in PXE ${ }^{[4]}$. Our patient had a history of chronic asymmetric left lower extremity oedema, probably associated with vascular disease, as well as the recent episode of ischaemic stroke. In addition, she had had HTA and progressive loss of visual accuracy. Kidney eGFR was normal (>90 ml/min). On the contrary, laboratory findings suggested a mineral and bone disorder (hypercalcaemia, hypophosphataemia, elevated parathyroid hormone levels and hypercalciuria), probably associated with hyperparathyroidism. Of note, patients suffering from renal disease show these alterations when eGFR falls below $60 \mathrm{ml} / \mathrm{min}$. Thus, the main explanations for these abnormalities are probably more related to primary hyperparathyroidism and/or PXE than the mild renal disease observed. Moreover, the albuminuria observed in our patient may represent an expression of vascular damage rather than the consequence of elevated blood pressure.

To the best of our knowledge, the main expressions of kidney disease in patients with PXE are nephrocalcinosis, glomerulonephritis, renovascular hypertension, ureteral disruption and/or recurrent urinary tract infection ${ }^{[3]}$. Additionally, some cases of nephrolithiasis associated with PXE have been reported.

Following our evaluation, the patient was started on cinacalcet $30 \mathrm{mg}$ daily for treatment of hyperparathyroidism and losartan was switched to olmesartan $40 \mathrm{mg} / \mathrm{day}$ for better control of blood pressure. In this regard, the use of calcimimetics in PXE patients with non-uremic calciphylaxis has been reported. Cinacalcet directly blocks PTH secretion by the parathyroid gland and indirectly lowers serum calcium levels. Thus, once PTH and calcium levels recover, hypercalciuria and hypophosphataemia are corrected.

In conclusion, this is an interesting presentation of classical renal manifestations in a patient with mild renal dysfunction. Given the impact of this disease, nephrologists may seek additional supportive features to improve and avoid the risk of complications. Moreover, PXE per se represents an interesting model to evaluate vascular disease in the early stages of renal disease.

\section{REFERENCES}

1. Neldner KH. Pseudoxanthoma elasticum. Clin Dermatol 1988;6(1):1-159.

2. Li Q, van de Wetering K, Uitto J. Pseudoxanthoma elasticum as a paradigm of heritable ectopic mineralization disorders: pathomechanisms and treatment development. Am J Pathol 2019;189(2):216-225.

3. Lau WL, Liu S, Vaziri ND. Chronic kidney disease results in deficiency of ABCC6, the novel inhibitor of vascular calcification. Am J Nephrol 2014;40(1):51-55.

4. Mendelsohn G, Bulkley BH, Hutchins GM. Cardiovascular manifestations of pseudoxanthoma elasticum. Arch Pathol Lab Med 1978;102(6):298-302. 